\title{
Effect of Aerobic Training on Cognitive Function and Arterial Stiffness in Sedentary Young Adults: A Pilot Randomized Controlled Trial
}

\author{
Samuel Asamoah, ${ }^{1}$ Jason Siegler, ${ }^{1}$ Dennis Chang, ${ }^{1,2}$ Andrew Scholey, ${ }^{3}$ \\ Alan Yeung, ${ }^{2}$ and Birinder S. Cheema ${ }^{1}$ \\ ${ }^{1}$ School of Science and Health, University of Western Sydney, Campbelltown, NSW 2650, Australia \\ ${ }^{2}$ Centre for Complementary Medicine Research, University of Western Sydney, Campbelltown, NSW 2650, Australia \\ ${ }^{3}$ Centre for Human Psychopharmacology, Swinburne University, Melbourne, VIC 3122, Australia \\ Correspondence should be addressed to Birinder S. Cheema; b.cheema@uws.edu.au
}

Received 12 October 2012; Revised 12 December 2012; Accepted 26 December 2012

Academic Editor: Germán Vicente-Rodriguez

\begin{abstract}
Copyright (C) 2013 Samuel Asamoah et al. This is an open access article distributed under the Creative Commons Attribution License, which permits unrestricted use, distribution, and reproduction in any medium, provided the original work is properly cited.

This study measured cognitive and vascular responses to aerobic training in sedentary young adults. Ten adults (6 women, 4 men; 18-29 years) were randomly assigned to an experimental or no-treatment control group. The experimental group engaged in a 6week intervention, performed on exercise cycle and treadmill, 3x/week, $50 \mathrm{~min} / \mathrm{session}$; intensity was increased over time. Outcome measures included arterial stiffness (augmentation index, $\mathrm{AIx}$, and pulse pressure), cardiorespiratory fitness $\left(\mathrm{VO}_{2 \max }\right)$, and cognitive function (attention, processing speed, working memory, episodic memory, and executive function). Participants randomized to aerobic training improved processing speed versus control $(P=0.02, \mathrm{ES}=0.55)$. However, no group $\times$ time effects were noted in other domains of cognitive function. AIx was reduced by approximately $16 \%$ from before to after intervention in the experimental group; however, the improvement was not statistically significant versus control $(P=0.20, \mathrm{ES}=0.22)$. Pulse pressure did not change between groups over time $(P=0.97, \mathrm{ES}=0.0)$. $\mathrm{VO}_{2 \max }$ increased by approximately $10 \%$ in the experimental group; however, the change was not significant between groups over time $(P=0.16, \mathrm{ES}=0.27)$. Vascular and cognitive adaptations to aerobic training may move in parallel. Robust trials simultaneously investigating a broad spectrum of aerobic training interventions and vascular and cognitive outcomes are warranted.
\end{abstract}

\section{Introduction}

Aging is associated with neurocognitive decline, which can lead to mild cognitive impairment (MCI) and dementia such as Alzheimer's disease [1]. Epidemiological data have shown that higher levels of physical activity [2-4] and fitness [58] are associated with better cognitive function and protect against cognitive decline [9]. Moreover, a recent metaanalysis of 29 randomized controlled trials involving healthy and chronically diseased adults of all ages, including individuals with MCI, concluded that aerobic training for more than one month can significantly improve measures of cognitive function, including attention, processing speed, memory, and executive function (i.e., cognitive flexibility) [10]. Such findings have led to a general consensus that exercise can enhance and/or maintain cognitive vitality throughout the lifespan [11]. Accordingly, exercise interventions to prevent cognitive decline should be initiated or maintained in early adulthood when participation in strenuous physical activities is often markedly reduced [12].

Measures of arterial stiffness (e.g., augmentation index, pulse pressure) increase as a consequence of aging and chronic disease due to the fragmenting and splitting of elastin fibres caused by mechanical stress $[13,14]$. Evidence suggests that arterial stiffness is increased in MCI and dementia [15, 16]. Moreover, arterial stiffness is strongly associated with atherosclerosis [17], and the relationship between cardiovascular disease and cognitive impairment 
is well established [18, 19]. Mitchell et al. [13] recently noted that increased arterial stiffness, evaluated via arterial tonometry, is concomitant with smaller brain tissue volumes and poorer cognitive performance in nondemented adults. Pase et al. [16] have shown that increased pulse pressure and augmentation index are associated with poorer memory and attention in apparently healthy middle-aged adults. Hence, arterial stiffness is associated with anatomical and functional deterioration of the brain $[13,16,20,21]$. Studies have consistently shown that measures of arterial stiffness, evaluated by means of various noninvasive methodologies, are inversely proportional to measures of cognitive function $[13,21-23]$.

To date, there has been limited exploration of the vascular changes that underlie or accompany the exercise-induced improvement of cognitive function. Aerobic training has been shown to improve arterial stiffness in young adults [24] and those with chronic cardiometabolic diseases [2527]. This improvement of arterial stiffness, reflecting an improvement of the endothelium, may be one potential mechanism by which cognitive function can be enhanced. However, to our knowledge, no randomized controlled trial has simultaneously measured vascular and cognitive adaptations to aerobic training in an attempt to elucidate this link in sedentary young adults, a cohort who may be predisposed to cognitive impairment later in life.

The purpose of this pilot study was to investigate the effect of a 6-week aerobic training intervention on markers of cognitive function and arterial stiffness in a cohort of sedentary young adults (aged 18-29 years). We hypothesized that aerobic training would significantly improve measures of cognitive function, including attention and processing speed, working memory, episodic memory, and executive function and that these changes would be concomitant with an improvement in arterial stiffness and cardiorespiratory fitness.

\section{Methods}

2.1. Study Design. This pilot study followed a parallel group design comparing the outcomes from participants assigned to an experimental treatment group (aerobic training) with those assigned to a no-treatment control group. Measures relating to cognitive and cardiovascular function were collected prior to and following a 6-week intervention period. Participants were randomly assigned via computer-generated blocks stratified by gender. An investigator not involved in data collection prepared the assignments, which were delivered to participants in sealed envelopes upon the completion of baseline testing. The university ethics committee approved all research procedures, and written informed consent was received from all participants.

2.2. Participants. All participants were recruited by means of flyer advertisements.

Eligibility Criteria. Men and women aged 18-29 years, sedentary (i.e., engaging in moderate-intensity exercise fewer than three days per week for 30 min per session); willingness
TABle 1: Computerised Mental Performance Assessment System (COMPASS) tests administered for the assessment of five cognitive function domains.

\begin{tabular}{|c|c|}
\hline Cognitive function domain & COMPASS tests \\
\hline \multirow{2}{*}{ Attention and processing speed } & Choice reaction time \\
\hline & Rapid visual information processing \\
\hline \multirow{2}{*}{ Working (short-term) memory } & N-back (3-back) \\
\hline & Immediate word recall \\
\hline \multirow{3}{*}{ Episodic (long-term) memory } & Delayed word recall \\
\hline & Delayed word recognition \\
\hline & Delayed picture recognition \\
\hline Executive function & Card sorting task \\
\hline
\end{tabular}

to exercise three sessions per week; no use of stimulants and/or medications known to acutely influence cardiovascular or cognitive function (e.g., ginkgo, ginseng, marijuana, alcohol abuse); no acute or chronic medical conditions that would make exercise potentially hazardous or primary outcomes impossible to assess as outlined by the American College of Sports Medicine [28]; ability to communicate in English; and willingness to provide written informed consent.

2.3. Aerobic Training Group. Participants randomly assigned to the aerobic training group engaged in a supervised sixweek intervention where they attended three sessions per week, approximately 50 min per session. Participants exercised for 20 minutes on each of two ergometers: (1) Reebok Performance B5.1e Upright Bike (Reebok, Essex, UK) and (2) Johnson JET 6000 treadmill (Johnson Health Tech. Co. Ltd). Training intensity for each participant was determined using the maximal heart rate $\left(\mathrm{HR}_{\max }\right)$ obtained during the baseline assessment of $\mathrm{VO}_{2 \text { max }}$. Initial training intensity was set at $65 \% \mathrm{HR}_{\max }( \pm 5$ beats per minute), increased by $5 \%$ per week to $80 \% \mathrm{HR}_{\max }$ ( \pm 5 beats per minute), and then was maintained at $80 \% \mathrm{HR}_{\max }$ from week 4 to 6 of the intervention. Heart rate was monitored throughout the training sessions via Polar Heart Rate monitors (Polar Electro FT4, Kempele, Finland). Each training session involved a $5 \mathrm{~min}$ warm-up and $5 \mathrm{~min}$ cool-down period.

2.4. Control Group. Participants randomised into the control group received no exercise intervention and were encouraged to maintain their normal lifestyle habits.

2.5. Outcome Measures. Outcome measures were collected in all participants prior to and following the 6-week intervention period at week 0 and week 7 , respectively. Postintervention testing in the aerobic training group was completed 48-96 hours after the completion of the final exercise bout to allow for adequate recovery and to ensure that changes were independent of acute effects. The week-7 measures were collected at the same time of day and same day of the week as baseline tests in order to control for the confounding effects of circadian rhythm. 


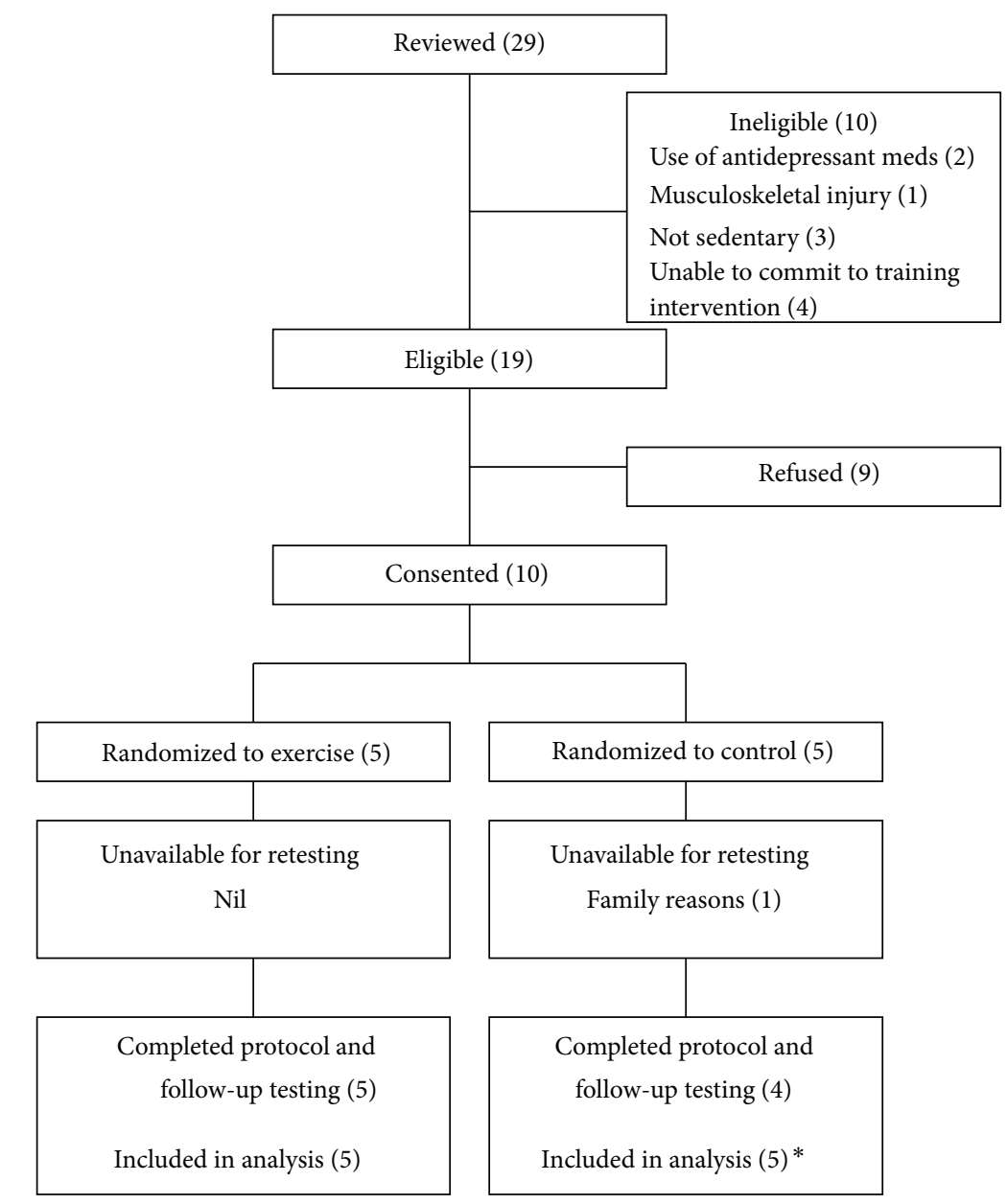

* Baseline data carried forward for one participant unavailable for follow-up testing

FIgURE 1: Flow of participants.

TABLE 2: Participant characteristics at baseline.

\begin{tabular}{|c|c|c|c|c|c|c|}
\hline \multirow{2}{*}{ Characteristic } & \multicolumn{3}{|c|}{ Control group $(n=5)$} & \multicolumn{3}{|c|}{ Aerobic training group $(n=5)$} \\
\hline & Mean \pm SD & Min & Max & Mean \pm SD & Min & Max \\
\hline Age, $y$ & $22.6 \pm 3.8$ & 20.0 & 29.0 & $23.8 \pm 3.6$ & 20.0 & 28.0 \\
\hline Height, $\mathrm{cm}$ & $166.8 \pm 7.4$ & 158.9 & 177.0 & $167.9 \pm 8.3$ & 160.0 & 182.0 \\
\hline Weight, kg & $66.4 \pm 12.9$ & 56.9 & 88.9 & $71.0 \pm 18.7$ & 49.1 & 96.2 \\
\hline BMI, $\mathrm{kg} \cdot \mathrm{m}^{-2}$ & $23.9 \pm 4.2$ & 19.7 & 30.2 & $24.9 \pm 4.9$ & 17.9 & 29.8 \\
\hline Waist circ., $\mathrm{cm}$ & $77.4 \pm 8.1$ & 71.0 & 90.5 & $81.8 \pm 15.6$ & 65.5 & 99.0 \\
\hline SBP, mmHg & $109.3 \pm 4.6$ & 105.0 & 116.0 & $118.2 \pm 12.8$ & 106.0 & 133.0 \\
\hline DBP, mmHg & $68.8 \pm 6.1$ & 61.0 & 77.0 & $71.8 \pm 4.2$ & 68.0 & 79.0 \\
\hline
\end{tabular}

Data reported as mean \pm standard deviation.

Min: minimum value; Max: maximum value; BMI: Body Mass Index; Waist circ: waist circumference; SBP: Systolic blood pressure; DBP: Diastolic blood pressure.

2.6. Cognitive Function. The Computerised Mental Performance Assessment System (COMPASS) (Brain, Performance and Nutrition Research Centre, Northumbria University, UK) was used to evaluate five major domains of cognitive function, including (1) attention, (2) processing speed, (3) working (short-term) memory, (4) episodic (long-term) memory, and (5) executive function. The specific tests used to evaluate each domain (Table 1) have been extensively used and validated [29-32]. The methodological procedure for each test is presented in the appendix 1. Aggregate scores 
TABLE 3: Summary of outcome measures.

\begin{tabular}{|c|c|c|c|c|c|c|}
\hline \multirow{2}{*}{ Outcome Measure } & \multicolumn{2}{|c|}{ Control $(n=5)$} & \multicolumn{2}{|c|}{ Exercise $(n=5)$} & \multirow{2}{*}{ Effect Size } & \multirow{2}{*}{$P$ value } \\
\hline & Before & After & Before & After & & \\
\hline \multicolumn{7}{|l|}{ Cognitive measures: } \\
\hline Attention (\%) & $58.9 \pm 8.8$ & $60.5 \pm 10.1$ & $78.2 \pm 17.2$ & $76.6 \pm 16.8$ & .00 & .87 \\
\hline Processing Speed (ms) & $463.3 \pm 23.5$ & $532.3 \pm 78.8$ & $410.9 \pm 49.0$ & $381.4 \pm 52.5$ & .55 & .02 \\
\hline Working Memory (\%) & $62.7 \pm 3.0$ & $56.9 \pm 10.8$ & $68.7 \pm 12.3$ & $65.6 \pm 10.2$ & .08 & .45 \\
\hline Episodic Memory (\%) & $71.3 \pm 4.4$ & $66.2 \pm 7.2$ & $81.1 \pm 9.7$ & $79.3 \pm 10.7$ & .08 & .47 \\
\hline Executive function ( $n$ of errors) & $20.2 \pm 6.4$ & $15.6 \pm 8.8$ & $9.2 \pm 2.3$ & $8.2 \pm 2.2$ & .01 & .81 \\
\hline Augmentation Index (\%) & $-9.8 \pm 7.7$ & $-8.2 \pm 8.8$ & $-6.2 \pm 5.7$ & $-8.8 \pm 12.6$ & .22 & .20 \\
\hline Aortic Pulse Pressure (mmHg) & $23.2 \pm 4.1$ & $25.8 \pm 1.9$ & $26.0 \pm 5.1$ & $26.2 \pm 4.4$ & .00 & .97 \\
\hline Aortic SBP (mmHg) & $92.4 \pm 4.5$ & $96.8 \pm 8.87$ & $99.0 \pm 8.6$ & $94.8 \pm 8.2$ & .39 & .07 \\
\hline Aortic DBP (mmHg) & $69.2 \pm 6.5$ & $71.0 \pm 9.6$ & $73.0 \pm 4.6$ & $68.4 \pm 5.1$ & .25 & .17 \\
\hline Brachial HR (beats/min) & $70.4 \pm 14.9$ & $70.0 \pm 10.3$ & $74.0 \pm 10.3$ & $63.8 \pm 6.1$ & .23 & .19 \\
\hline Brachial SBP (mmHg) & $109.3 \pm 4.6$ & $114.0 \pm 7.9$ & $118.2 \pm 12.8$ & $112.6 \pm 12.1$ & .25 & .17 \\
\hline Brachial DBP (mmHg) & $68.8 \pm 6.1$ & $70.1 \pm 9.3$ & $71.8 \pm 4.2$ & $67.4 \pm 5.2$ & .21 & .21 \\
\hline $\mathrm{VO}_{2 \max }\left(\mathrm{mL} / \mathrm{kg}^{-1} / \mathrm{min}^{-1}\right)$ & $35.1 \pm 6.3$ & $35.2 \pm 7.8$ & $33.8 \pm 7.3$ & $37.2 \pm 7.1$ & .27 & .16 \\
\hline
\end{tabular}

Data reported as mean \pm standard deviation.

SBP: Systolic blood pressure; DBP: diastolic blood pressure; HR: heart rate.

for attention, working memory, and episodic memory were obtained by averaging the scores for the tests assessing each domain (Table 1). Scores for each test were obtained as the percentage of correct responses; higher values denoted better cognitive function. Processing speed was measured in milliseconds (ms) with lower scores denoting faster processing speed; aggregate scores were obtained by averaging the scores for the two tests assessing this domain (Table 1). Executive function was scored by total sum of errors on the card sorting task with lower scores representing better executive function. We employed a familiarisation session $\geq 24$ hours prior to baseline testing to introduce, explain, and trial the tests of cognitive function with our participants. This session also served to minimize practice effects.

2.7. Arterial Stiffness and Hemodynamic Measures. Participants were instructed to avoid food and beverages for a minimum of 3 hours prior to testing. All measurements were collected with participants in a supine position. Resting blood pressures and heart rate were evaluated in a supine position following $10 \mathrm{~min}$ supine rest using an automated cuff (HEM-7221, Omron Inc., Japan) positioned on the arm (i.e., brachial artery). The average of three recordings for each measure was recorded. Measures of arterial stiffness (i.e., central pulse pressure and augmentation index) were obtained using the SphygmoCor System (AtCor Medical Pty, Sydney, Australia) and hand-held, high-fidelity tonometer (Millar Instruments, Houston, Texas) at the radial artery. This system has the ability to derive the central aortic pressure waveform noninvasively from the pressure pulse recorded at a peripheral site. The aortic pressure waveforms are comprised of a forward wave caused by left ventricular contraction and a reflected wave due to the backflow arising from regions of increased impedance in the peripheral vessels. Pulse pressure was automatically calculated by deducting the central diastolic pressure from the central systolic pressure. Augmentation index (AIx), representing the extent to which the aortic pressure is augmented by reflected pressure waves, was calculated by dividing the augmentation pressure by the pulse pressure, multiplied by 100 . Given AIx is affected by factors such as ejection fraction and heart rate [33], the SphygmoCor algorithm normalizes AIx to a heart rate of 75 beats per minute. This method is highly reproducible [34] and has been used extensively to evaluate arterial stiffness in healthy $[24,35]$ and chronically diseased cohorts [27]. Population norms for AIx range from $-23.27 \%$ to $63.07 \%$ in adults (aged 18-86 years) with larger values indicating greater arterial stiffness [36].

2.8. Cardiorespiratory Fitness. Cardiorespiratory fitness $\left(\mathrm{VO}_{2 \text { max }}\right)$ was assessed at baseline (week 0) and followup (week 7) to evaluate the effectiveness of the exercise intervention. $\mathrm{VO}_{2 \max }$ was assessed via indirect calorimetry (Jaeger Metabolic Gas Analysers, Viasys Healthcare, Germany) using a standard ramp protocol employed on a laboratory treadmill (LE 200 CE, Viasys Health Care, USA). Before each test, $\mathrm{O}_{2}$ and $\mathrm{CO}_{2}$ sensors were calibrated using high-grade calibration gas with certified gas concentrations $\left(\mathrm{O}_{2}=16 \%, \mathrm{CO}=5 \%, \mathrm{~N}_{2}=\right.$ balance $)$. Ventilation volume was measured using a "Triple V" Digital Flow Meter and calibrated using a $3 \mathrm{~L}$ calibration syringe (Hans Rudolph, Kansas, MO, USA). The ramp protocol started at either 5.0 or $7.0 \mathrm{~km} \cdot \mathrm{hr}^{-1}$ (depending on fitness) then increased by $1 \mathrm{~km} \cdot \mathrm{hr}^{-1}$ each minute for a total of five minutes, whereby the grade was increased $2 \%$ every minute until volitional fatigue. $\mathrm{VO}_{2 \max }$ was defined as the attainment of a plateau $\left(<2 \mathrm{~mL} \cdot \mathrm{kg}^{-1} \cdot \mathrm{min}^{-1}\right.$ increase $)$ or decline in oxygen consumption with an increase in workload over the final minute, the attainment of age-predicted maximal heart rate $\left( \pm 10 \mathrm{~b} \cdot \mathrm{min}^{-1}\right)$ of the conventional age-predicted maximum 
using the Inbar equation [37], and a respiratory exchange ratio (RER) of $>1.15$.

2.9. Demographics and Health Status. Demographic and health status data were collected during the screening process and baseline testing by means of standard questionnaires and assessments. Factors included demographic and morphometric data, including age, gender, height, weight, waist circumference, and concurrent medication, and supplement usage and dosage.

2.10. Statistical Analyses. Analyses were performed using the Statistical Package for the Social Sciences (IBM, SPSS Version 19.0). All data were inspected visually and statistically for normality (skewness and kurtosis between -1 and +1 ). All available data were included regardless of participant compliance to the aerobic exercise intervention and analyses were completed according to intention-to-treat strategy, using the last-observation-carry-forward imputation method for any missing data. Changes between the experimental and control group were determined by analysis of covariance (ANCOVA) of the posttreatment score controlling for the baseline score. A $P$ value of $<0.05$ was considered indicative of statistical significance.

\section{Results}

3.1. Flow of Participants. Twenty-nine individuals expressed an interest in participating in the trial (Figure 1). Ten were excluded because they did not meet the entry criteria, while nine elected not to consent. Ten participants ( 6 women and 4 men) consented and were randomly assigned to the exercise or control group, only one participant was unavailable for follow-up testing.

3.2. Baseline Characteristics. Baseline characteristics for the control group and the aerobic training group are presented in Table 2. No significant differences were detected between groups at baseline according to these descriptive characteristics (all $P>0.05$ ). Gender was equally distributed between the two groups (i.e., three women and two men in each group). The cohort ranged in age from 20 to 29 years; all participants were university students. One participant in the control group was diagnosed with and medicated for anxiety, while another participant in the control group was medicated for hypercholesterolemia in week 5 of the intervention period. One participant in the control group fulfilled the clinical criteria for obesity (BMI $>30 \mathrm{~kg} / \mathrm{m}^{2}$ ), while three in the aerobic training group and one in the control group fulfilled the clinical criteria for overweight $\left(\right.$ BMI $\left.>25 \mathrm{~kg} / \mathrm{m}^{2}\right)$. None of the participants had diabetes, or a history or current use of cigarettes. All participants were normotensive at baseline and none were medicated for hypertension.

3.3. Compliance and Adverse Events. Compliance to the intervention was high at $84.4 \pm 14.4 \%$ in the five participants assigned to the aerobic training group. No adverse events were reported or documented during the trial.

3.4. Outcome Measures. All outcome measures are presented in Table 3. The aerobic training group significantly reduced processing speed when compared to that of the control group $(P=0.02, \mathrm{ES}=0.55)$. However, no group $\times$ time effects were noted in the other domains of cognitive function, including attention $(P=0.87)$, working memory $(P=0.45)$, episodic memory $(P=0.47)$, and executive function $(P=0.81)$. The aerobic training reduced AIx by approximately $16 \%$ from before to after intervention; however, this improvement was not statistically significant versus the control group $(P=$ 0.20 , ES $=0.22$, Table 3$)$. Central pulse pressure did not change between groups over time $(P=0.97, \mathrm{ES}=0.0$, Table $3)$. However, central systolic $(P=0.07)$ and diastolic pressure $(P=0.17)$ showed trends toward improvement in the experimental versus control group (Table 3 ). No differences in resting brachial heart rate, diastolic or systolic blood pressure were detected between groups over time; however, direction of adaptation favoured the exercise group (Table 3 ). At baseline, $\mathrm{VO}_{2 \max }$ in both the experimental and control group was "poor" in relation to age-referenced normative data [38]. $\mathrm{VO}_{2 \max }$ increased by approximately $10 \%$ in the aerobic training group from week 0 to week 7 ; however, this change did not achieve statistical significance versus the control group $(P=0.16, \mathrm{ES}=0.27$, Table 3$)$.

3.5. Sample Size Calculation. A post hoc sample size calculation for arterial stiffness was computed using the noncommercial statistical power analysis program $G^{*}$ Power based on change score data for the aerobic training group $(-2.6 \pm 7.7 \%)$ and the control group $(+1.6 \pm 4.2 \%)$. Using a two-tailed test of significance with an alpha level of 0.05 , approximately 72 participants (36 per group) would provide $80 \%$ power to detect a statistically significant difference between groups on this outcome measure.

\section{Discussion}

This pilot study investigated the effect of a 6-week aerobic training intervention on cognitive function, arterial stiffness, and cardiorespiratory fitness in a cohort of sedentary young adults. Participants assigned to the experimental group experienced statistically significant improvements in processing speed versus the control group $(P=0.02)$. However, other measures of cognitive function, including attention, working memory, episodic memory, and executive function did not differ between groups over time. The improvement of processing speed in the aerobic training group was concomitant with positive but nonsignificant (group $\times$ time) adaptation of $\mathrm{VO}_{2 \max }(P=0.16)$ and AIx $(P=0.20)$. Central pulse pressure did not change between groups over time $(P=0.97$, ES $=0.0$, Table 3$)$. However, central systolic $(P=0.07)$ and diastolic pressure $(P=0.17)$ showed trends toward improvement in the experimental versus control group. We acknowledge that our findings are preliminary and recognize the need for large-scale, well-controlled trials investigating 
the vascular mechanisms that underlie the exercise-induced improvement of cognitive function.

Targeting sedentary behaviour during adolescence and young adulthood is imperative for averting chronic diseases and for promoting general physical and mental health and well-being throughout life. Notably, however, only a few trials have investigated the effect of prolonged aerobic training on measures of cognitive function in young populations [39-41] and none of these trials enrolled an entirely sedentary cohort. Interestingly, the majority of trials to date have investigated middle-aged and elderly cohorts [10,42, 43], individuals that may be well beyond the peak of cognitive health [44]. Ideally, effective preventive therapies such as aerobic exercise should be undertaken earlier in life.

In the present study, we noted an improvement of processing speed $(P=0.02)$ in our exercising participants. This is a novel finding in sedentary young adults, though a similar effect has been noted in older women [45]. The improvement of processing speed may be particularly important in young adults given that this measure is associated with higher intelligence quotient [46] and academic aptitude [47, 48]. Accordingly, meaningful improvements of processing speed could contribute to greater success in vocational and educational setting.

Other benefits of aerobic training have been noted in young adults. Stroth et al. [39] investigated the effect of an aerobic training intervention prescribed 3 sessions per week for 6 weeks in 28 young adults (17-29 years). Each session was 30 min duration and involved running (or briskly walking) at $70-90 \%$ of anaerobic threshold. At the end of the intervention period, the exercise group significantly improved cardiorespiratory fitness versus the control group $(P=0.04)$. This adaptation was accompanied by an increase in visuospatial memory $(P=0.01)$ and a trend toward improved attention/concentration $(P=0.08)$ and number of errors made during the test of attention/concentration $(P=0.15)$. The intervention, however, had no effect on verbal memory $(P=0.86)$. In a subsequent study, Stroth et al. [40] tested a similar running intervention for 17 weeks in 75 young adults (17-47 years) and found that significant improvements in cardiorespiratory fitness $(P<0.01)$ were concomitant with increases in executive function (i.e., cognitive flexibility) $(P<$ $0.05)$ and cognitive control $(P<0.05)$. Hansen et al. [41] also noted an improvements in executive function, as well as reaction time, in a trial prescribing eight weeks of aerobic training in 37 young sailors (18-22 years). Hence, there is some evidence to suggest that aerobic training improves measures of cognitive function in early adulthood.

Few studies to date have measured changes in arterial stiffness in the context of aerobic training in young adults [49-51]. This is notable given that asymptomatic vascular changes can manifest early in life $[52,53]$, while crosssectional studies have indeed demonstrated that endurancetrained young adults have lower levels of arterial stiffness than less active peers $[35,54]$. Cameron and Dart [50] noted significant improvements in systemic arterial compliance (i.e., reduced arterial stiffness) in 13 sedentary adults secondary to 4 weeks of aerobic training prescribed $1.5 \mathrm{hr} /$ session, 3 sessions/wk. This finding was supported by Kakiyama et al.
[51] who prescribed 8 weeks of aerobic training, 3-4 sessions per week at $70 \% \mathrm{VO}_{2 \max }$ for $60 \mathrm{~min} /$ session in 10 sedentary young males (19-24 years). Although our study showed no significant changes in AIx and pulse pressure, there are reasons to believe that aerobic exercise may improve arterial compliance, which is insensitive to AIx and pulse pressure measures. For example, Currie et al. [49] reported significant reductions in central and peripheral pulse wave velocity $(P<$ $0.05)$ with no change in AIx or pulse pressure in a study of 14 young males ( $25 \pm 4$ years) participating in a 6-day aerobic training intervention (2 hours per day at $65 \% \mathrm{VO}_{2 \max }$ ).

Evidence suggests that reductions in arterial stiffness in humans are influenced by improvements in endothelial function, which may be principally mediated by the increases in endothelial shear stress experienced during exercise [55]. Endothelial dysfunction is highly responsive to exercise intervention [55]. Several trials have noted that exercise training can improve endothelial dysfunction in young children and adolescents with obesity [56, 57], suggesting that arterial health can be improved early in life. Overall, the literature suggests that interventions that specifically target cardiorespiratory fitness may mitigate the stiffening of arteries which accompanies the aging process [58].

It is not known how improvements in arterial health correspond to improvements in cognition given that no other trials have investigated these outcomes simultaneously, to our knowledge. However, our findings and the studies we have reviewed suggest that vascular and cognitive measures may move in parallel in response to aerobic training intervention. Large-scale, longitudinal trials simultaneously investigating a broad spectrum of aerobic training interventions and pertinent vascular and cognitive outcomes are warranted. Trials should specifically be conducted in young adults who are physically inactive, given that this cohort is at risk of vascular and cognitive decline. Our post hoc sample size estimate suggests that 72 participants would be required to evaluate significant changes in AIx. Several cognitive outcomes, including attention, working memory, episodic memory, and executive function failed to change in the present study. However, longer and more frequent training could potentially influence these outcomes positively. Longer interventions and longer-term followup would be required of future trials. Methodologies utilising computed tomography, magnetic resonance imaging, flow-mediated dilation, and other measures of endothelial function should be utilized.

\section{Conclusion}

In summary, this pilot study demonstrates that sedentary young adults participating in a 6-week aerobic training intervention can experience statistically significant improvements in processing speed versus a nonexercising control group $(P=0.02)$. This improvement was accompanied by a positive but nonsignificant improvement of $\mathrm{VO}_{2 \max }(P=0.16)$ and arterial stiffness $(P=0.20)$. Continued investigation of the vascular mechanisms that underlie the exercise-induced improvement of cognitive function is required. 


\section{Appendix}

(1) Choice Reaction Time. In this study, an arrow pointing either left or right was presented on the monitor and participants were instructed to press either the " $Z$ " key for left or the "M" key for right as quickly and as accurately as possible. A total of 40 trials were presented with varying interstimulus intervals (one-to-three seconds) to reduce anticipation.

(2) Rapid Visual Information Processing. In this study, a series of digits ranging from 1 to 9 were presented one after the other with a stimulus presentation time of $500 \mathrm{~ms}$. Participants were required to press the "spacebar" whenever they detect three consecutive even (i.e., any three of $2,4,6,8$ ) or three consecutive odd (i.e., any three of $1,3,5,7,9)$ numbers in a row.

(3) N-back (3-back). A series of letters were displayed randomly in upper or lower case on the computer screen, one at a time. Participants were instructed to respond if the letter that appears was also presented 3 letters previously in the series. The letter(s) can be matched regardless of the case they appear in (e.g., G, y, h, g, k). Participants are required to press the $M$ key for "YES" and to press the Z key for "NO."

(4) Immediate Word Recall. Participants were presented with a list of 15 words to be remembered. These were presented on the monitor at a rate of one-word per second. Directly after word presentation, participants were given one minute to recall as many words as possible from the word presentation list.

(5) Delayed Word Recall. Participants were presented with a list of 15 words to be remembered. Approximately $20 \mathrm{~min}$ after word presentation, the participant was given 60 seconds to record on paper as many of the items from the original word presentation as possible.

(6) Delayed Word Recognition. Word recognition: following the delayed word recall, participants were presented with words from the original list, randomly scattered with an equal number of distracter words. Participants were required to decide whether the word presented on the screen was from the original word presentation or not by pressing the " $Z$ " key for NO and the "M" key for YES.

(7) Delayed Picture Recognition. Fifteen pictures of common items are presented on the computer screen. Each picture was displayed for duration of 3 seconds, with 1-second interstimulus duration. Picture recognition was tested by the presentation of the original items along with an equal number of distracters in random order. Participants were asked to respond by pressing the " $Z$ " key for NO and the " $M$ " key for YES to specify whether the picture(s) were presented previously.

(8) Card Sorting Task. participants were asked to match each card that appears at the bottom of the screen to one of the four piles (numbered 1, 2, 3,4) displayed in the upper part of the screen. The cards are matched by using the mouse to click on the pile to which the participant believes it belongs to. Cards were to be matched by either the colour, shape, or pattern displayed on the top pile of cards; however, participants are not told how to match the cards, but are told whether they are right or wrong each time. If the participant obtained an incorrect match, they were simply asked to try another matching technique to match the next card. There is no time limit on this task.

\section{References}

[1] J. E. Graham, K. Rockwood, B. L. Beattie et al., "Prevalence and severity of cognitive impairment with and without dementia in an elderly population," The Lancet, vol. 349, no. 9068, pp. 1793-1796, 1997.

[2] S. Rovio, I. Kåreholt, E.-L. Helkala et al., "Leisure-time physical activity at midlife and the risk of dementia and Alzheimer's disease," Lancet Neurology, vol. 4, no. 11, pp. 705-711, 2005.

[3] L. Fratiglioni, S. Paillard-Borg, and B. Winblad, "An active and socially integrated lifestyle in late life might protect against dementia," Lancet Neurology, vol. 3, no. 6, pp. 343-353, 2004.

[4] R. D. Abbott, L. R. White, G. W. Ross, K. H. Masaki, J. D. Curb, and H. Petrovitch, "Walking and dementia in physically capable elderly men," Journal of the American Medical Association, vol. 292, no. 12, pp. 1447-1453, 2004.

[5] R. Newson and E. B. Kemps, "Cardiorespiratory fitness as a predictor of successful cognitive ageing," Journal of Clinical and Experimental Neuropsychology, vol. 28, no. 6, pp. 949-967, 2006.

[6] J. M. Bugg, K. Shah, D. T. Villareal, and D. Head, "Cognitive and neural correlates of aerobic fitness in obese older adults," Experimental Aging Research, vol. 38, no. 2, pp. 131-145, 2012.

[7] S. Colcombe and A. F. Kramer, "Fitness effects on the cognitive function of older adults: a meta-analytic study," Psychological Science, vol. 14, no. 2, pp. 125-130, 2003.

[8] M. A. I. Åberg, N. L. Pedersen, K. Torén et al., "Cardiovascular fitness is associated with cognition in young adulthood," Proceedings of the National Academy of Sciences of the United States of America, vol. 106, no. 49, pp. 20906-20911, 2009.

[9] D. E. Barnes, K. Yaffe, W. A. Satariano, and I. B. Tager, "A longitudinal study of cardiorespiratory fitness and cognitive function in healthy older adults," Journal of the American Geriatrics Society, vol. 51, no. 4, pp. 459-465, 2003.

[10] P. J. Smith, J. A. Blumenthal, B. M. Hoffman et al., "Aerobic exercise and neurocognitive performance: a meta-analytic review of randomized controlled trials," Psychosomatic Medicine, vol. 72, no. 3, pp. 239-252, 2010.

[11] A. F. Kramer, K. I. Erickson, and S. J. Colcombe, "Exercise, cognition, and the aging brain," Journal of Applied Physiology, vol. 101, no. 4, pp. 1237-1242, 2006.

[12] P. Gordon-Larsen, M. C. Nelson, and B. M. Popkin, "Longitudinal physical activity and sedentary behavior trends: adolescence to adulthood," American Journal of Preventive Medicine, vol. 27, no. 4, pp. 277-283, 2004.

[13] G. F. Mitchell, M. A. van Buchem, S. Sigurdsson et al., "Arterial stiffness, pressure and flow pulsatility and brain structure and function: the age, gene/environment susceptibility-Reykjavik study," Brain, vol. 134, part 11, pp. 3398-3407, 2011.

[14] C. M. Chung, Y. S. Lin, C. M. Chu et al., "Arterial stiffness is the independent factor of left ventricular hypertrophy determined by electrocardiogram," The American Journal of the Medical Sciences, vol. 344, no. 3, pp. 190-193, 2012.

[15] O. Hanon, S. Haulon, H. Lenoir et al., "Relationship between arterial stiffness and cognitive function in elderly subjects with complaints of memory loss," Stroke, vol. 36, no. 10, pp. 2193-2197, 2005. 
[16] M. P. Pase, A. Pipingas, M. Kras et al., "Healthy middle-aged individuals are vulnerable to cognitive deficits as a result of increased arterial stiffness," Journal of Hypertension, vol. 28, no. 8, pp. 1724-1729, 2010.

[17] N. M. van Popele, D. E. Grobbee, M. L. Bots et al., "Association between arterial stiffness and atherosclerosis: the Rotterdam study," Stroke, vol. 32, no. 2, pp. 454-460, 2001.

[18] L. Zheng, W. J. Mack, H. C. Chui et al., "Coronary artery disease is associated with cognitive decline independent of changes on magnetic resonance imaging in cognitively normal elderly adults," Journal of the American Geriatrics Society, vol. 60, no. 3, pp. 499-504, 2012.

[19] D. Bos, M. W. Vernooij, S. E. Elias-Smale et al., "Atherosclerotic calcification relates to cognitive function and to brain changes on magnetic resonance imaging," Alzheimers Dement, vol. 8, no. 5, supplement, pp. 104-111, 2012.

[20] M. F. O’Rourke and M. E. Safar, "Relationship between aortic stiffening and microvascular disease in brain and kidney: cause and logic of therapy," Hypertension, vol. 46, no. 1, pp. 200-204, 2005.

[21] M. P. Pase, A. Herbert, N. A. Grima, A. Pipingas, and M. F. O'Rourke, "Arterial stiffness as a cause of cognitive decline and dementia: a systematic review and meta-analysis," Internal Medicine Journal, vol. 42, no. 7, pp. 808-815, 2012.

[22] S. W. Rabkin and G. Jarvie, "Comparison of vascular stiffness in vascular dementia, Alzheimer dementia and cognitive impairment," Blood Press, vol. 20, no. 5, pp. 274-283, 2011.

[23] H. Triantafyllidi, C. Arvaniti, J. Lekakis et al., "Cognitive impairment is related to increased arterial stiffness and microvascular damage in patients with never-treated essential hypertension," American Journal of Hypertension, vol. 22, no. 5, pp. 525-530, 2009.

[24] M. J. Goldberg, S. H. Boutcher, and Y. N. Boutcher, "The effect of 4 weeks of aerobic exercise on vascular and baroreflex function of young men with a family history of hypertension," Journal of Human Hypertension, vol. 26, no. 11, pp. 644-649, 2012.

[25] A. G. Huebschmann, W. M. Kohrt, and J. G. Regensteiner, "Exercise attenuates the premature cardiovascular aging effects of type 2 diabetes mellitus," Vascular Medicine, vol. 16, no. 5, pp. 378-390, 2011.

[26] K. M. Madden, C. Lockhart, D. Cuff, T. F. Potter, and G. S. Meneilly, "Short-term aerobic exercise reduces arterial stiffness in older adults with type 2 diabetes, hypertension, and hypercholesterolemia," Diabetes Care, vol. 32, no. 8, pp. 1531-1535, 2009.

[27] S. Mustata, C. Chan, V. Lai, and J. A. Miller, "Impact of an exercise program on arterial stiffness and insulin resistance in hemodialysis patients," Journal of the American Society of Nephrology, vol. 15, no. 10, pp. 2713-2718, 2004.

[28] W. Thompson, N. Gordon, and L. Pescatello, Eds., ACSM'S Guidelines for Exercise Testing and Prescription, Wolters Kluwer/Lippincott Williams \& Wilkins, Philadelphia, Pa, USA, 2010.

[29] R. C. Gur, J. Richard, P. Hughett et al., "A cognitive neuroscience-based computerized battery for efficient measurement of individual differences: standardization and initial construct validation," Journal of Neuroscience Methods, vol. 187, no. 2, pp. 254-262, 2010.

[30] M. J. Kane, A. R. A. Conway, T. K. Miura, and G. J. H. Colflesh, "Working memory, attention control, and the n-back task: a question of construct validity," Journal of Experimental Psychology, vol. 33, no. 3, pp. 615-622, 2007.
[31] K. W. Greve, F. Ingram, and K. J. Bianchini, "Latent structure of the Wisconsin Card Sorting Test in a clinical sample," Archives of Clinical Neuropsychology, vol. 13, no. 7, pp. 597-609, 1998.

[32] E. A. Berg, "A simple objective technique for measuring flexibility in thinking," The Journal of General Psychology, no. 39 , p. $15,1948$.

[33] J. E. Sharman, J. E. Davies, C. Jenkins, and T. H. Marwick, "Augmentation index, left ventricular contractility, and wave reflection," Hypertension, vol. 54, no. 5, pp. 1099-1105, 2009.

[34] I. B. Wilkinson, S. A. Fuchs, I. M. Jansen et al., "Reproducibility of pulse wave velocity and augmentation index measured by pulse wave analysis," Journal of Hypertension, vol. 16, no. 12, part 2, pp. 2079-2084, 1998.

[35] D. G. Edwards and J. T. Lang, "Augmentation index and systolic load are lower in competitive endurance athletes," American Journal of Hypertension, vol. 18, no. 5, pp. 679-683, 2005.

[36] "Reference values for SphygmoCor Px," http://www.atcormedical.com/pdf/TN8\%20\%20Reference\%20Values\%20for\%20SphygmoCor\%20Px.pdf.

[37] R. A. Robergs and R. Landwehr, "The surprising history of the "HRmax = 220-age" equation," Journal of Exercise Physiology Online, vol. 5, no. 2, pp. 1-10, 2002.

[38] V. Heywood, Advanced Fitness Assessment and Exercise Prescription, Human Kinetics, Champaign, Ill, USA, 5th edition, 2006.

[39] S. Stroth, K. Hille, M. Spitzer, and R. Reinhardt, "Aerobic endurance exercise benefits memory and affect in young adults," Neuropsychological Rehabilitation, vol. 19, no. 2, pp. 223-243, 2009.

[40] S. Stroth, R. K. Reinhardt, J. Thöne et al., "Impact of aerobic exercise training on cognitive functions and affect associated to the COMT polymorphism in young adults," Neurobiology of Learning and Memory, vol. 94, no. 3, pp. 364-372, 2010.

[41] A. L. Hansen, B. H. Johnsen, J. J. Sollers, K. Stenvik, and J. F. Thayer, "Heart rate variability and its relation to prefrontal cognitive function: the effects of training and detraining," European Journal of Applied Physiology, vol. 93, no. 3, pp. 263-272, 2004.

[42] P. Heyn, B. C. Abreu, and K. J. Ottenbacher, "The effects of exercise training on elderly persons with cognitive impairment and dementia: a meta-analysis," Archives of Physical Medicine and Rehabilitation, vol. 85, no. 10, pp. 1694-1704, 2004.

[43] P. C. Heyn, K. E. Johnson, and A. F. Kramer, "Endurance and strength training outcomes on cognitively impaired and cognitively intact older adults: a meta-analysis," Journal of Nutrition, Health and Aging, vol. 12, no. 6, pp. 401-409, 2008.

[44] T. A. Salthouse, "When does age-related cognitive decline begin?" Neurobiology of Aging, vol. 30, no. 4, pp. 507-514, 2009.

[45] R. E. Rikli and D. J. Edwards, "Effects of a three-year exercise program on motor function and cognitive processing speed in older women," Research Quarterly for Exercise and Sport, vol. 62, no. 1, pp. 61-67, 1991.

[46] P. A. Vernon, "Speed of information processing and general intelligence," Intelligence, vol. 7, no. 1, pp. 53-70, 1983.

[47] S. J. Cohn, J. S. Carlson, and A. R. Jensen, "Speed of information processing in academically gifted youths," Personality and Individual Differences, vol. 6, no. 5, pp. 621-629, 1985.

[48] P. A. Vernon and A. R. Jensen, "Individual and group differences in intelligence and speed of information processing," Personality and Individual Differences, vol. 5, no. 4, pp. 411-423, 1984. 
[49] K. D. Currie, S. G. Thomas, and J. M. Goodman, "Effects of short-term endurance exercise training on vascular function in young males," European Journal of Applied Physiology, vol. 107, no. 2, pp. 211-218, 2009.

[50] J. D. Cameron and A. M. Dart, "Exercise training increases total systemic arterial compliance in humans," American The Journal of Physiology, vol. 266, no. 2, pp. H693-H701, 1994.

[51] T. Kakiyama, M. Matsuda, and S. Koseki, "Effect of physical activity on the distensibility of the aortic wall in healthy males," Angiology, vol. 49, no. 10, pp. 749-757, 1998.

[52] E. M. Tuzcu, S. R. Kapadia, E. Tutar et al., "High prevalence of coronary atherosclerosis in asymptomatic teenagers and young adults evidence from intravascular ultrasound," Circulation, vol. 103, no. 22, pp. 2705-2710, 2001.

[53] L. T. Mahoney, T. L. Burns, W. Stanford et al., "Coronary risk factors measured in childhood and young adult life are associated with coronary artery calcification in young adults: the Muscatine study," Journal of the American College of Cardiology, vol. 27, no. 2, pp. 277-284, 1996.

[54] I. Ferreira, J. W. R. Twisk, C. D. A. Stehouwer, W. van Mechelen, and H. C. G. Kemper, "Longitudinal changes in VO2max: associations with carotid IMT and arterial stiffness," Medicine and Science in Sports \& Exercise, vol. 35, no. 10, pp. 1670-1678, 2003.

[55] D. J. Green, A. Maiorana, G. O'Driscoll, and R. Taylor, "Effect of exercise training on endothelium-derived nitric oxide function in humans," The Journal of Physiology, vol. 561, no. 1, pp. 1-25, 2004.

[56] A. S. Kelly, R. J. Wetzsteon, D. R. Kaiser, J. Steinberger, A. J. Bank, and D. R. Dengel, "Inflammation, insulin, and endothelial function in overweight children and adolescents: the role of exercise," The Journal of Pediatrics, vol. 145, no. 6, pp. 731-736, 2004.

[57] J. H. Park, M. Miyashita, Y. C. Kwon et al., "A 12-week after-school physical activity programme improves endothelial cell function in overweight and obese children: a randomised controlled study," BMC Pediatrics, vol. 12, no. 1, p. 111, 2012.

[58] P. V. Vaitkevicius, J. L. Fleg, J. H. Engel et al., "Effects of age and aerobic capacity on arterial stiffness in healthy adults," Circulation, vol. 88, no. 4, pp. 1456-1462, 1993. 

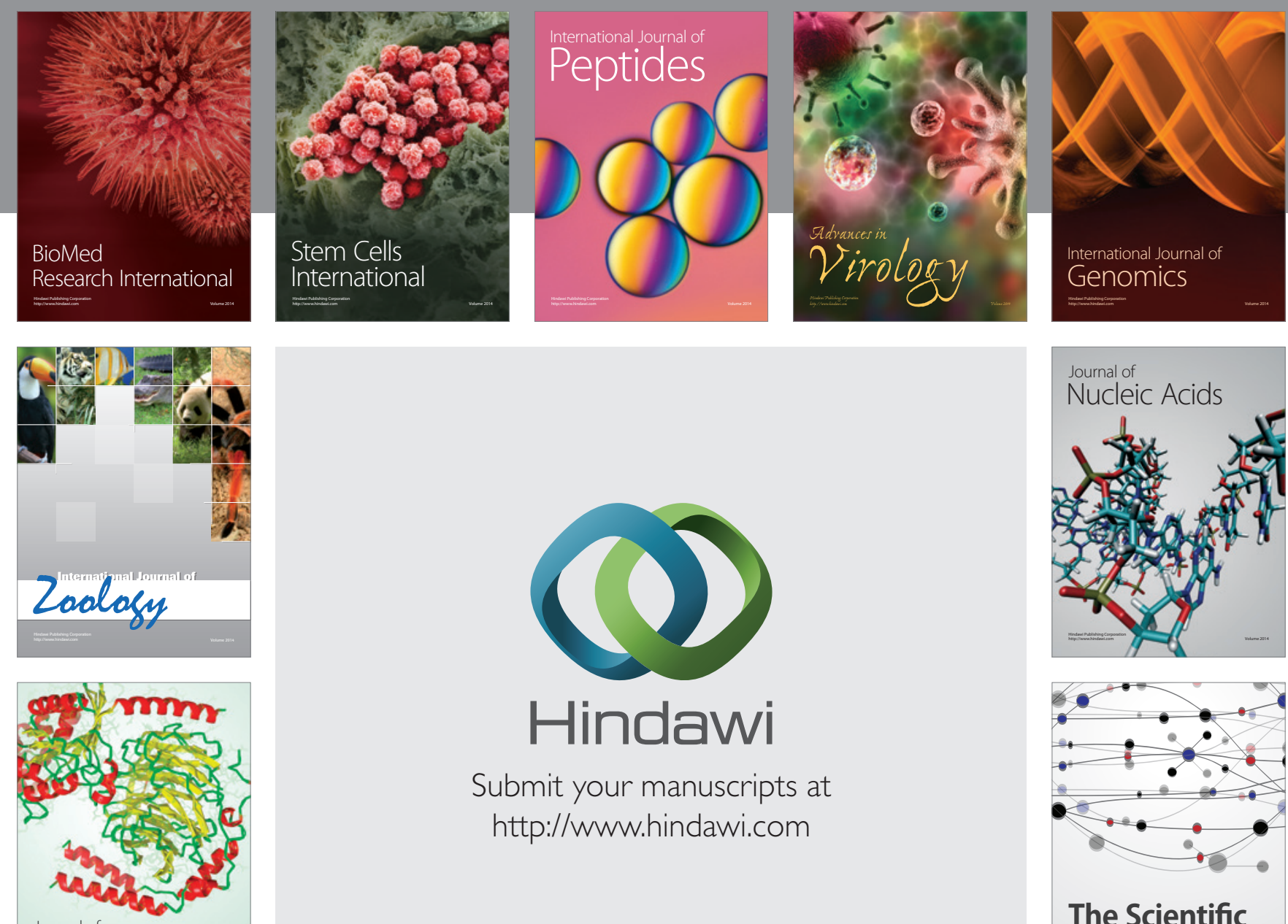

Submit your manuscripts at

http://www.hindawi.com

Journal of
Signal Transduction
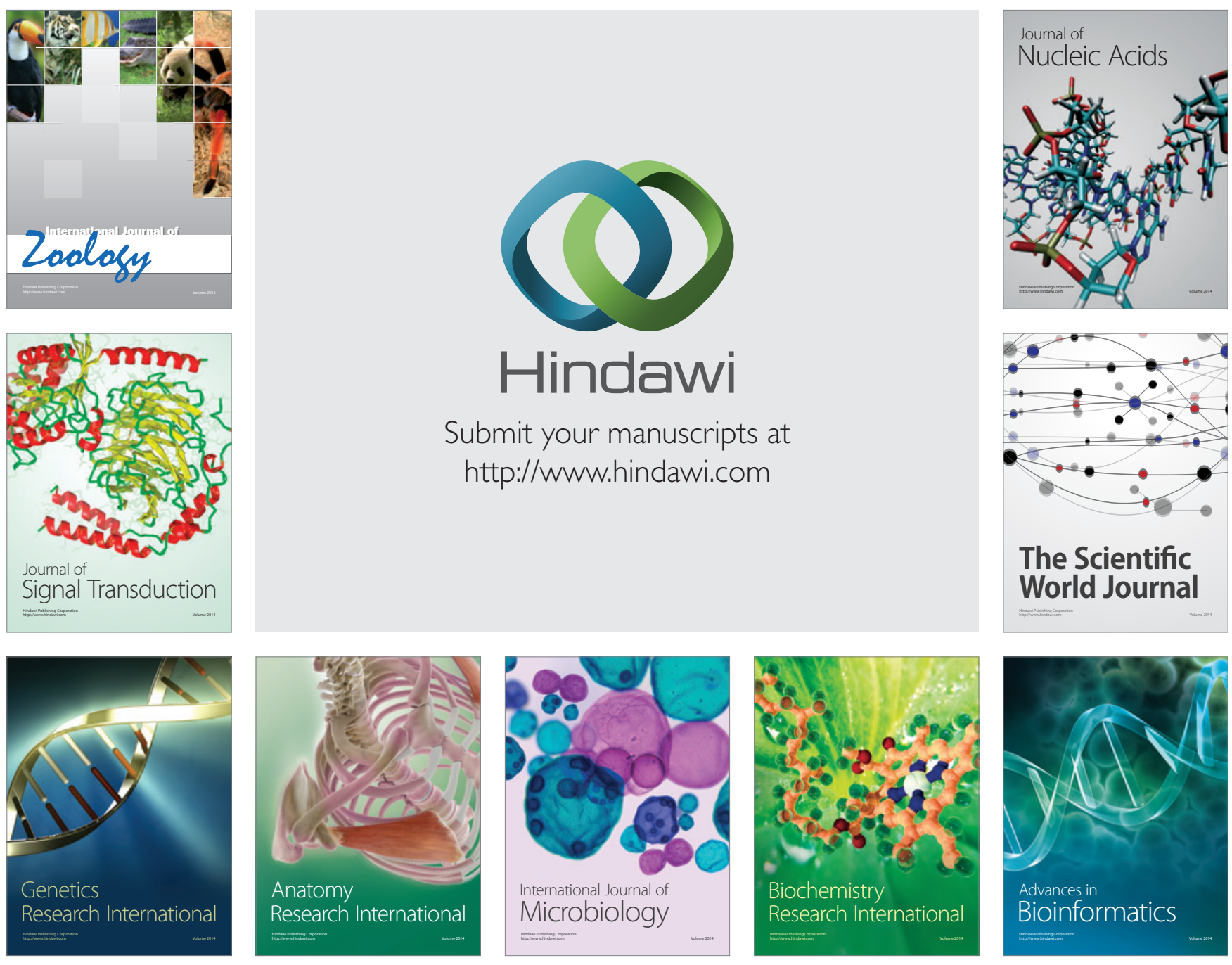

The Scientific World Journal
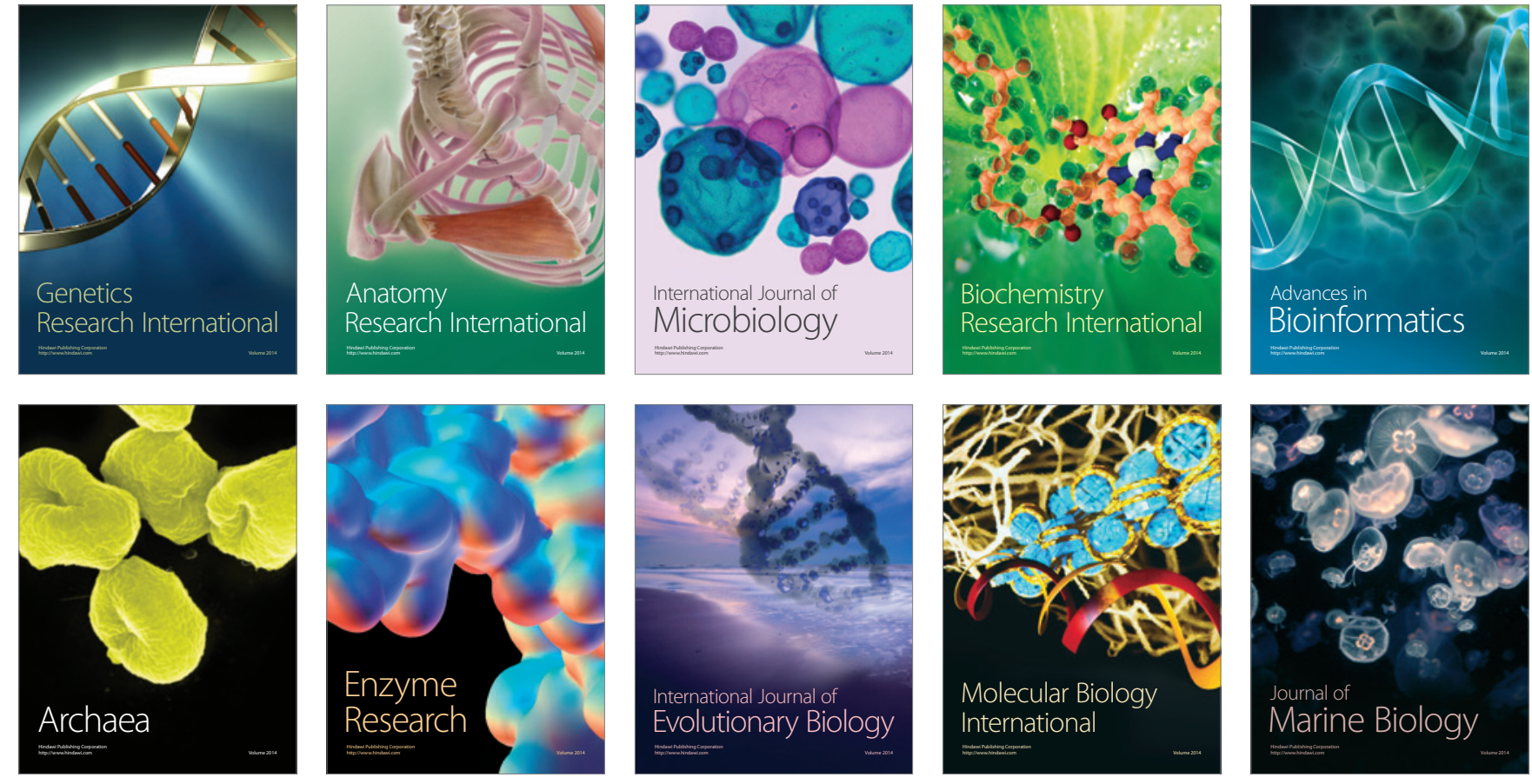